\title{
Frequency Domain Identification and Identifiability Analysis of a Nonlinear Vehicle Drivetrain Model
}

\author{
Eduard Popp ${ }^{1}$, Mathias Tantau ${ }^{1}$, Mark Wielitzka ${ }^{1}$, Tobias Ortmaier ${ }^{1}$, and Dennis Giebert ${ }^{2}$
}

\begin{abstract}
Physical parameters of a vehicle drivetrain are required in many applications. In the context of fault diagnosis, for example, knowledge about the installed components or parts can provide insights in order to verify if they behave in accordance with standards or deviate from them in a way that adversely affects the operating performance. For this purpose a frequency domain identification approach is presented, which is based only on standard mounted sensors. In the presented method in particular nonlinear effects are taken into account resulting from backlash. In order to guarantee a unique parameter set a local identifiability analysis is performed. The main idea of the method is to exploit the dependency between the frequency response of the nonlinear system and the magnitude of the test-signal to improve optimization of the physical parameters. Finally, identification results using real measurement data are presented.
\end{abstract}

\section{INTRODUCTION}

Model-based approaches are being given increasingly attention in the field of automotive engineering and are used for manifold applications and assignments. In this term simulation models are used e.g. for controller design, fault detection and diagnosis, condition monitoring, and onlineparameter estimation in order to obtain the longitudinal road gradient or road friction coefficient [1]-[4]. For this purpose accurate models are required. Special challenges arise in particular during parameter identification. Since the installation of additional sensors lead to further effort, only standard sensors are available. Hence, the identification process becomes more complex. Regarding grey-box models, the confidence in the identified physical parameters must be validated for the given measurements and procedure in order to judge the usability of the results for model-based application.

The vehicle drivetrain contains all components from the engine to the tires. In this paper, the physical parameters of a vehicle drivetrain are to be identified using only the torque and the averaged engine speed when the vehicle is at idle-speed, the transmission is in neutral gear, and the clutch is engaged. Hence, the drivetrain contains the dual mass flywheel (DMF), the rotating parts of the gearbox and the engine, which is considered separately. The installed DMF is a nonlinear component with multi-level springs, backlash and dry friction [5]. In the past two decades the system's dynamics of the DMF have already been investigated in detail. Accurate nonlinear models are found in [5], [6], that show very good agreement in a wide range from idle-speed

\footnotetext{
${ }^{1}$ Institute of Mechatronic Systems, Leibniz Universität Hannover, Germany eduard.poppeimes. uni-hannover. de

${ }^{2}$ IAV Automotive Engineering
}

up to $5000 \mathrm{~min}^{-1}$. The DMFs were measured on a test bench with precisely provided torque on the drive and load sides, as well as angles and angular velocity measurements. However, for identification purpose on production vehicles these models are not usable, as the solver require step size about $100 \mu$ s and due to a lack of sensors. Time domain identification methods of systems with backlash have been described in [7], [8]. However, these require at least the measurement of the engine side angular position, which is not available in this case. Further challenges are motorexcited vibrations that superimpose with the engine speed. Frequency domain methods, described in [9], [10], point out the influence of backlash on the frequency response, but don't use it for identification. Therefore this paper presents a frequency-based approach in which two chirp signals of different amplitudes are used. Since the operating point has an influence on the system response as described in section II-B, the identification is carried out in closed-loop at almost constant speed. Within the framework of an identifiability analysis, the presented identification procedure is finally evaluated using a simulation example and real measurement data.

The paper is organized as follows. In section II the closedloop model within the nonlinear drivetrain model and engine dynamics is introduced. In section III the frequency domain identification approach is presented. The identification analysis follows in section IV and the results with real measurement data are discussed in section V. Finally, a conclusion is given in section VI.

\section{MODELING}

For the purpose of identification the closed-loop system is introduced in Fig. 1. The idle-speed controller provides a torque request of the combustion engine in order to adjust the idle-speed $n_{\text {setpoint }}$. Adding the generated test-signal $T_{\text {test }}$ for excitation of the drivetrain, the total torque request $T_{\mathrm{ecu}}$ is formed. The applied torque $T_{\text {eng }}$ with mean-value torque $T_{\mathrm{m}}$ (see section II-A) may vary according to $T_{\text {ecu }}$ depending on how well the engine is calibrated in certain operating point. The measured engine speed $\tilde{n}$ using a speed sensor is obtained in order to close the loop. Furthermore cylinder pressure sensors are given to reconstruct the mean-value torque $T_{\mathrm{m}}$. For the results within this paper each cylinder is equipped with a sensor. In case of a single sensor, the reconstruction can be replaced by model-based methods that are state of the art [11]. In the following sections the briefly introduced components are described in detail, as they have been implemented in Matlab/SIMULINK. 


\section{A. Engine dynamics}

The engine torque $T_{\text {eng }}$ consists of the combustion torque $T_{\mathrm{cmb}}$, the compression and expansion torque $T_{\mathrm{c} / \mathrm{e}}$ and the mass torque $T_{\mathrm{j}}$. As they arise from non-continuous combustion and non-linear torque transmission on the crankshaft drive, they depend on the angular position $\varphi_{1}$ and speed of the crankshaft $\omega_{1}$.

$$
T_{\mathrm{eng}}=\sum_{i=1}^{N_{\mathrm{cyl}}} T_{\mathrm{cmb}}\left(\varphi_{1, i}\right)+T_{\mathrm{c} / \mathrm{e}}\left(\varphi_{1, i}\right)+T_{\mathrm{j}}\left(\varphi_{1, i}, \omega_{1, i}\right)
$$

In order to approximate the combustion torque $T_{\mathrm{cmb}}$ of a single cylinder a Fourier-series describing a rect-function can be used [12]

$$
\begin{aligned}
T_{\mathrm{cmb}}= & \frac{T_{\mathrm{m}}}{N_{\mathrm{cyl}}}\left(1+\sum_{k=0.5}^{4} \frac{8}{\pi k} \sin \left(k \frac{\pi}{4}\right) \sin \left(k \varphi_{1}+\phi_{k}\right)\right) \\
& \text { with } k=0.5 ; 1 ; 1.5 ; \ldots ; 4 \\
& \text { and } \phi_{k}=\frac{\pi}{2}\left(1-\frac{k}{2}\right) .
\end{aligned}
$$

The applied mean-value engine torque $T_{\mathrm{m}}$ according to one working cycle of $4 \pi \mathrm{rad}$ is a nonlinear function of many variables, such as fuel mass $\dot{m}_{\mathrm{f}}$, air/fuel ratio $\lambda_{\mathrm{a} / \mathrm{f}}$, engine speed $\dot{\varphi}_{1}$, EGR rate $x_{\text {egr }}$ etc. [13]. Depending on how well the combustion engine is calibrated, the mean-value engine torque follows the requested torque $T_{\mathrm{ecu}}$ appropriately

$$
T_{\mathrm{m}}=T_{\mathrm{ecu}} \cdot f\left(\dot{m}_{\mathrm{f}}, \lambda_{\mathrm{a} / \mathrm{f}}, \dot{\varphi}_{1}, x_{\mathrm{egr}}, \ldots\right) .
$$

For a perfectly calibrated engine the nonlinear function is equal to 1 for all operating points. Using the cross-sectional cylinder area $A$, manifold pressure $p_{1}$, the maximum cylinder volume $V_{\mathrm{z}}$, the minimum cylinder volume $V_{\mathrm{c}}$, the isentropic exponent $\kappa$, the cylinder position $s$ and velocity $\dot{s}$, which depend on the crankshaft position $\varphi, T_{\mathrm{c} / \mathrm{e}}$ can be expressed as follows

$$
T_{\mathrm{c} / \mathrm{e}}=A p_{\mathrm{l}}\left(\frac{V_{\mathrm{z}}}{V_{\mathrm{c}}+A s\left(\varphi_{1}\right)}\right)^{\kappa} \dot{s}(\varphi)
$$

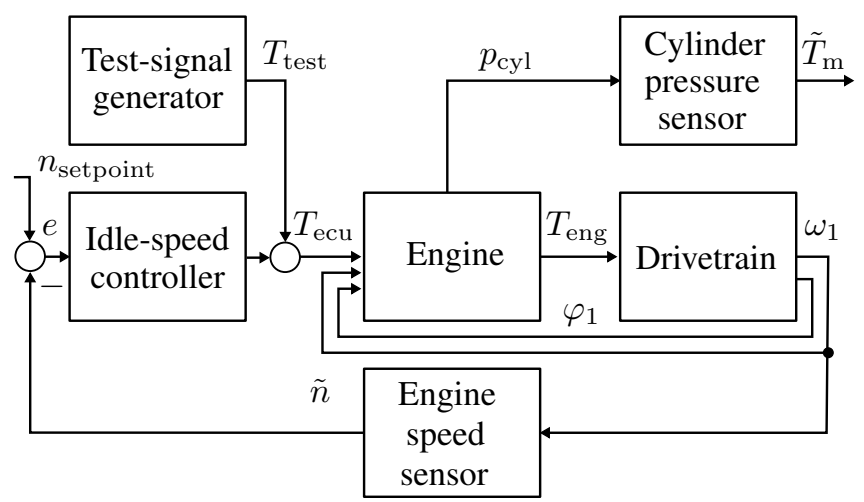

Fig. 1: Closed-loop system consisting of four-clyinder fourstroke combustion engine, the drivetrain, a speed sensor, cylinder pressure sensors, idle-speed controller and a testsignal generator.
At last the mass torque $T_{\mathrm{j}}$ follows the equation

$$
\begin{aligned}
T_{\mathrm{j}}= & m_{\mathrm{osc}} r^{2} \omega_{1}^{2}\left(\frac{\lambda_{\mathrm{P}}}{4} \sin \left(\varphi_{1}\right)-\frac{1}{2} \sin (2 \varphi)\right. \\
& \left.-\frac{3 \lambda_{\mathrm{P}}}{4} \sin \left(3 \varphi_{1}\right)-\left(\frac{\lambda_{\mathrm{P}}}{2}\right)^{2} \sin \left(4 \varphi_{1}\right)\right)
\end{aligned}
$$

where the crank radius $r$, the ratio of the crank radius to connecting rod length $\lambda_{P}=r / l$ are denoted. The mass of the piston and approximately $1 / 3$ of the total connecting rod mass is contained in $m_{\mathrm{osc}}$.

\section{B. Drivetrain dynamics}

In this paper only a good approximation of the dynamic behavior at idle-speed is decisive. For this purpose the drivetrain is assumed as three mass system shown in Fig. 2, which is based on [6] and [14]. Nevertheless there are other model structures conceivable, that also lead to a frequency response similar to Fig. 3. For the given there are some simplifications carried out. First, the multistage arc springs are replaced by springs with constant stiffness $c_{12}$, since the identification takes place at constant speed. The backlash $\varepsilon_{12}$ arises as the arc spring is shorter than its tunnel. The DMF furthermore exhibits coulomb friction characteristic, which is represented by $T_{\mathrm{c}, 12}$. As the clutch is engaged $\varepsilon_{23}$ denotes backlash in the gear and the clutch hub. Torque losses $T_{\text {loss }}$ are assumed to be constant at idle-speed. This leads to the equation of motion:

$$
\begin{aligned}
J_{1} \ddot{\varphi}_{1}= & T_{\text {eng }}-T_{\text {loss }} \\
& -c_{12}\left(\delta_{12}-\varepsilon_{12}\right)-d_{12} \dot{\delta}_{12}-T_{\mathrm{c}, 12} \operatorname{sign}\left(\dot{\delta}_{12}\right) \\
J_{2} \ddot{\varphi}_{2}= & c_{12}\left(\delta_{12}-\varepsilon_{12}\right)+d_{12} \dot{\delta}_{12}+T_{\mathrm{c}, 12} \operatorname{sign}\left(\dot{\delta}_{12}\right) \\
& -\left(c_{23}\left(\delta_{23}-\varepsilon_{23}\right)+d_{23}\left(\dot{\delta}_{23}-\dot{\varepsilon}_{23}\right)\right. \\
J_{3} \ddot{\varphi}_{3}= & c_{23}\left(\delta_{23}-\varepsilon_{23}\right)+d_{23}\left(\dot{\delta}_{23}-\dot{\varepsilon}_{23}\right)
\end{aligned}
$$

The deflection is defined as $\delta_{12}=\varphi_{1}-\varphi_{2}$ and $\delta_{23}=\varphi_{2}-\varphi_{3}$, respectively its time derivative. The backlash is described introducing the states $\varepsilon_{12}$ and $\varepsilon_{23}$ :

$$
\varepsilon= \begin{cases}\varepsilon_{\max }, & \delta \geq \varepsilon_{\max } \\ \delta, & \varepsilon_{\min }<\delta<\varepsilon_{\max } \\ \varepsilon_{\min }, & \delta \leq \varepsilon_{\min }\end{cases}
$$

In case of gear play a physical model according to $\dot{\varepsilon}_{23}$ is used, which can be reference in [15]. Due to crankshaft based discrete events, the model is solved in angle discrete sampling using a angle transformation, as [6] suggested in

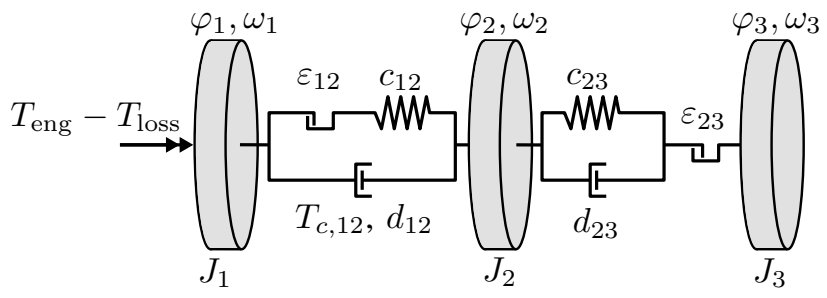

Fig. 2: Model structure of the drivetrain. 
terms of calculation efficiency. A step size of $\phi_{\mathrm{s}}=\frac{\pi}{30} \mathrm{rad}$ is set. At idle-speed of $n=1000 \mathrm{~min}^{-1}$ the sample time is $1 \mathrm{~ms}$ corresponding to following equation

$$
T_{\mathrm{s}}=\frac{\phi_{\mathrm{s}}}{\omega_{1}}=\frac{\phi_{\mathrm{s}}}{n} \frac{30}{\pi} .
$$

Therefore, a sample time $T_{\mathrm{S}}$ in the following refers to an angle step size $\phi_{\mathrm{S}}$ according to the current engine speed $n$.

\section{Sensors}

The engine speed measurement is performed using a mean average filter in order to filter engine induced oscillations of the second order. As its oscillation period is given by half crankshaft rotation $\phi_{2 o}=\pi$, there are $N_{\text {win }}=30$ data points to be averaged. The discrete frequency response at $n$ is

$$
\left|G_{\mathrm{n}}(j \omega)\right|=\frac{1}{N_{\text {win }}}\left|\frac{\sin \left(\omega / 2 T_{\mathrm{s}} N_{\text {win }}\right)}{\sin \left(\omega / 2 T_{\mathrm{s}}\right)}\right| \frac{30}{\pi},
$$

with circular frequency $\omega$. The filtered engine speed $\tilde{n}$ is sampled with $\phi_{\mathrm{s}, \mathrm{n}}=\pi / 2$. The mean-valued torque $\tilde{T}_{\mathrm{m}}$ can only be measured at working stroke. Hence, for a single cylinder $T_{\mathrm{m}}$ is sampled with $8 \phi_{\mathrm{s}, \mathrm{n}}$. Using four cylinder pressure sensors in total the sampling is performed with $2 \phi_{\mathrm{s}, \mathrm{n}}$. According to $\tilde{n}$ the torque needs to be up-sampled about factor $L=2$ by holding the last value. This interpolation method complies with the convolution with a rect-function in time-domain. In frequency-domain it can be expressed as follows (see [16])

$$
\begin{aligned}
\left|G_{\mathrm{p}}(j \omega)\right| & =\frac{1}{L}\left|\frac{\sin \left(\omega / 2 T_{\mathrm{s}, \mathrm{p}} L\right)}{\sin \left(\omega / 2 T_{\mathrm{s}, \mathrm{p}}\right)}\right|, \\
T_{\mathrm{s}, \mathrm{p}} & =\frac{\phi_{\mathrm{s}, \mathrm{n}}}{n} \frac{30}{\pi}=\frac{15}{n},
\end{aligned}
$$

using the sample time $T_{\mathrm{s}, \mathrm{p}}$.

\section{IDENTIFICATION METHOD}

In order to estimate the frequency response of the drivetrain a test-signal is added to the output of the idle-speed controller according to Fig. 1. This approach can be found in related experiments [9], [10]. In this paper the system is excited using a chirp signal with duration $T_{\mathrm{D}}$, starting frequency $f_{1}$, final frequency $f_{2}$ and amplitude $A_{\text {test }}$ :

$$
T_{\text {test }}=A_{\text {test }} \sin \left(2 \pi\left(\frac{1}{2}\left(f_{2}-f_{1}\right) \frac{t}{T_{\mathrm{D}}}+f_{1}\right) t\right) .
$$

The frequency response $G_{\mathrm{xy}}(j \omega)$ is determined by estimation of the power spectral density (PSD) $\phi_{\mathrm{xx}}(\Omega)$ and $\phi_{\mathrm{xy}}(\Omega)$ of the input $x(t)$ and output $y(t)$, that are sampled with $T_{\mathrm{s}}$

$$
\begin{aligned}
r_{x x}(k) & =\frac{1}{N} \sum_{n=1}^{N-1} x_{n} x_{n+k}, \\
\phi_{x x}(\Omega) & =\mathcal{F}\left\{r_{x x}(k)\right\}, \\
r_{x y}(k) & =\frac{1}{N} \sum_{n=1}^{N-1} x_{n} y_{n+k}, \\
\phi_{x y}(\Omega) & =\mathcal{F}\left\{r_{x y}(k)\right\}, \\
G_{\mathrm{xy}}(j \omega) & =\frac{T_{\mathrm{s}} \phi_{x y}(\Omega)}{T_{\mathrm{s}} \phi_{x x}(\Omega)}=\frac{S_{x y}(j \omega)}{S_{x x}(j \omega)},
\end{aligned}
$$

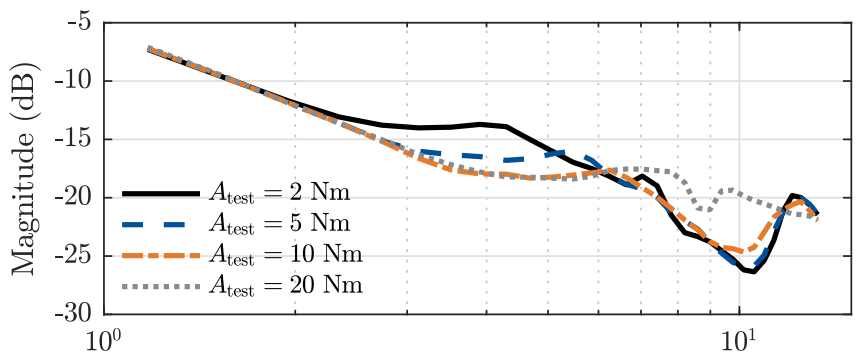

Frequency $(\mathrm{Hz})$

Fig. 3: Measurement data of the frequency response using a test-signal with different amplitudes.

using the Fourier-Transformation of the auto-correlation $r_{x x}(k)$ and cross-correlation $r_{x y}(k)$. The estimation can be improved by means of the Welch-method [17]. The measurement data is decomposed into various overlapped segments, so that the PSD is estimated from the averaged segments. As the identification is performed in closed-loop, , the input $x(t)$ is correlated with disturbances, that interfere with the feedback $y(t)$. In order to obtain the correct frequency response of the plant the following equation is applied

$$
G_{x y}(j \omega)=\frac{S_{z y}(j \omega)}{S_{z x}(j \omega)}
$$

using the test-signal $z(t)$. In our case $x(t)$ is $\tilde{T}_{\mathrm{m}}(t), y(t)$ is $\tilde{n}(t)$ and $z(t)$ is $T_{\text {test }}(t)$. Considering $G_{\mathrm{n}}$ and $G_{\mathrm{p}}$, the dynamic behaviour of the drivetrain and the engine is calculated as follows

$$
\begin{aligned}
\left|G_{\mathrm{DRV}}(j \omega)\right| & =\left|G_{\tilde{T}_{\mathrm{m}} \tilde{n}}(j \omega)\right|\left|G_{\mathrm{p}}(j \omega)\right|\left|G_{\mathrm{n}}(j \omega)\right|^{-1} \\
\left|G_{\mathrm{ENG}}(\omega)\right| & =\left|G_{T_{\mathrm{ecu}} \tilde{T}_{\mathrm{m}}}(j \omega)\right|\left|G_{\mathrm{p}}(j \omega)\right|^{-1}
\end{aligned}
$$

In order to obtain the parameters $\theta$, the frequency response $\left|G_{\mathrm{DRV}}(j \omega)\right|$ using measurement data and $\left|\tilde{G}_{\mathrm{DRV}}(\omega, \boldsymbol{\theta})\right|$ corresponding to model predicted data are calculated following eq. (14). The objective function to be minimized in frequency domain is defined as normalized root mean squared error

$$
\begin{aligned}
\mathrm{NRMSE} & =\frac{\| \zeta(\omega)-\tilde{\zeta}(\omega, \boldsymbol{\theta})) \|_{2}}{\left\|\zeta(\omega)-\frac{1}{N} \sum_{i=1}^{N} \zeta\left(\omega_{i}\right)\right\|_{2}} . \\
\zeta(\omega) & =20 \log \left(\left|G_{\mathrm{DRV}}(j \omega)\right|\right), \\
\tilde{\zeta}(\omega, \boldsymbol{\theta}) & =20 \log \left(\left|\tilde{G}_{\mathrm{DRV}}(j \omega, \boldsymbol{\theta})\right|\right) .
\end{aligned}
$$

In case of using two measurements with different amplitudes the errors are added

$$
\operatorname{NRMSE}=\operatorname{NRMSE}\left(A_{\text {test }, 1}\right)+\operatorname{NRMSE}\left(A_{\text {test }, 2}\right) .
$$

Fig. 3 shows the dynamic behaviour of $G_{\mathrm{DRV}}$ with different amplitudes $A_{\text {test }}$ of the test-signal. As discussed in section IV-A, the lower resonance frequency is left shifted for small amplitudes $A_{\text {test }}$. This effect can be assigned to backlash, which is discussed in the next section. 


\section{IDENTIFIABILITY ANALYSIS}

Repeating an optimization algorithm several times using random initial parameter values and all else unchanged may lead to different results for each repetition. In order to avoid such case, the identifiability has to be ensured. Following a general definition by [18], model parameters $\boldsymbol{\theta}$ are identifiable, if their estimates $\hat{\boldsymbol{\theta}}$ converge to the true values $\boldsymbol{\theta}_{\mathbf{0}}$. In this context there is a distinction between structural and practical identifiability. Using a minimal set of independent parameters to describe the equations of motion of a system guarantees structural identifiability. Otherwise there are redundant parameters. It is a property of the system equations [18], while practical identifiability takes into account the quality of the excitation signal and the amount of measured data. An introduction with further details can be found in [19]. By means of a reduced model structural identifiability analysis is performed using an analytical approach and the concept of profile likelihood [20] in order to illustrate the benefit of the identification method that is introduced in this paper.

\section{A. Analytic approach}

First, a simple dual mass system is considered with additional gain parameter $K$ acting as engine dynamics at a quasi-stationary operating point:

$$
G(s)=\frac{K}{s\left(J_{1}+J_{2}\right)} \frac{1+s \frac{d}{c}+s^{2} \frac{J_{2}}{c}}{1+s \frac{d}{c}+s^{2} \frac{J_{1} J_{2}}{\left(J_{1}+J_{2}\right) c}} .
$$

Regarding the bodeplot the magnitude is uniquely described by minimal parameters, given by the asymptote for low frequencies $G(s \rightarrow 0)$, high frequencies $G(s \rightarrow \infty)$, the natural frequency $\omega_{0(D)}$ and damping ratio $D_{(D)}$ of the denominator and numerator $\omega_{0(N)}, D_{(N)}$. According to [21] these characteristics can be calculated as follows, using the total mass $J_{\Sigma}=J_{1}+J_{2}$ and the mass ratio $x=\frac{J_{1}}{J_{\Sigma}}$ :

$$
\begin{aligned}
G(s \rightarrow 0) & =\frac{1}{s} \frac{K \alpha}{J_{\sum \alpha}}=\frac{1}{s} \frac{K^{*}}{J_{\sum}^{*}} \\
G(s \rightarrow \infty) & =\frac{1}{s x} \frac{K \alpha}{J_{\sum} \alpha}=\frac{1}{s x} \frac{K^{*}}{J_{\sum}^{*}} \\
\omega_{0(D)} & =\sqrt{\frac{1}{1-x} \frac{c \alpha}{J_{\sum} \alpha}}=\sqrt{\frac{1}{1-x} \frac{c^{*}}{J_{\sum}^{*}}} \\
D_{(D)} & =\frac{1}{2} \sqrt{\frac{1}{1-x} \frac{(d \alpha)^{2}}{J_{\sum} \alpha c \alpha}}=\frac{1}{2} \sqrt{\frac{1}{1-x} \frac{\left(d^{*}\right)^{2}}{J_{\sum}^{*} c^{*}}} \\
\omega_{0(N)} & =\omega_{0(D)} \sqrt{\frac{1}{x}}, \quad D_{(N)}=D_{(D)} \sqrt{\frac{1}{x}}
\end{aligned}
$$

In order to check, if there is an unambiguous assignment between characteristics and the physical parameter, the real parameters $\boldsymbol{\theta}=\left\{J_{\sum}, c, d, K\right\}$ are scaled by $\alpha$. Since $\boldsymbol{\theta}$ can be substituted by $\boldsymbol{\theta}^{*}$ without changing the characteristics, there is a correlation between them. Only parameter $x$ is structurally identifiable in this identification setup. It can therefore be assumed, that a structural identification of the drivetrain and the engine dynamics at the same time is not

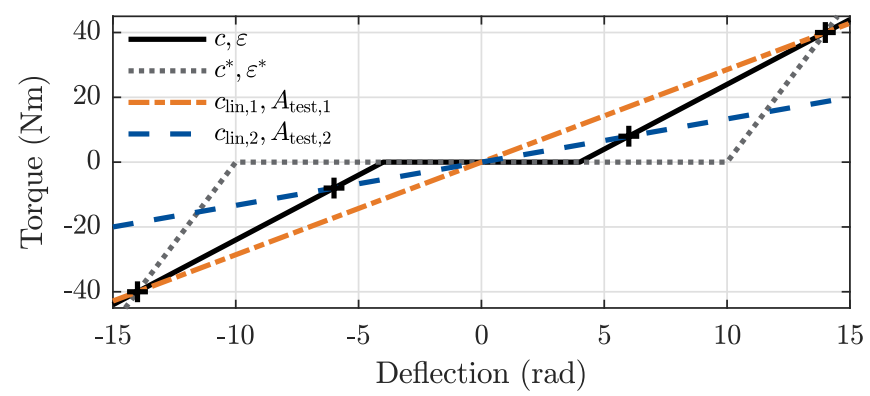

Fig. 4: Deflection of an undamped system with backlash. The black solid line shows the behaviour with parameter $c$ and $\varepsilon$. Using two sinusoidal test-signals in respect to $A_{\text {test, } 1}$ and $A_{\text {test,1 }}$ leads to a unique result.

possible. Next, backlash is added to the system. At this point an analytically based answer on structural identifiability is not easy to give. Nevertheless, a statement can be made for the undamped case. Using a sinusoidal torque test-signal with amplitude $A_{\text {test }}$ a certain deflection

$$
\delta=\frac{A_{\text {test }}}{c}
$$

occurs (see Fig. 4). The deflection is increased about $\varepsilon$ in case of additional backlash. A linearized model at this operating point shows a decreased spring stiffness

$$
c_{\text {lin }}=\frac{A_{\text {test }}}{\delta+\varepsilon}=\frac{A_{\text {test }}}{A_{\text {test }}+\varepsilon c} c
$$

This leads to a left shifted natural frequency. However, $\varepsilon$ and $c$ cannot be determined uniquely from $c_{\text {lin }}$ and $A_{\text {test }}$. Hence, for a given $c^{*}$ there is a

$$
\varepsilon^{*}=\left(\frac{1}{c_{\text {lin }}}-\frac{1}{c^{*}}\right) A_{\text {test }} .
$$

Thus, a further correlation is pointed out for $\{c, \varepsilon\}$ using a certain $A_{\text {test }}$. In order to obtain a unique result the identification should include at least two different excitation amplitudes. Using eq. (22) with $A_{\text {test }, 1}$ and $A_{\text {test,2, }}$, there is a unique solution

$$
\begin{aligned}
\left(\frac{1}{c_{\operatorname{lin}, 1}}-\frac{1}{c}\right) A_{\text {test }, 1} & =\left(\frac{1}{c_{\operatorname{lin}, 2}}-\frac{1}{c}\right) A_{\text {test }, 2} \\
\frac{A_{\text {test }, 1}}{A_{\text {test }, 2}} & =\frac{\left(c-c_{\operatorname{lin}, 2}\right) c_{\operatorname{lin}, 1}}{\left(c-c_{\operatorname{lin}, 1}\right) c_{\operatorname{lin}, 2}} \\
c & =\frac{c_{\text {lin }, 1} c_{\operatorname{lin}, 2}\left(A_{\text {test }, 1}-A_{\text {test }, 2}\right)}{A_{\text {test }, 1} c_{\text {lin }, 2}-A_{\text {test }, 2} c_{\text {lin }, 1}}
\end{aligned}
$$

This relation is illustrated in Fig. 4. In the following subsection, the identifiability of the system using the likelihood approach is regarded in order to verify the assumptions. First, a brief introduction is given.

\section{B. Profile likelihood approach}

According to [20] the main idea behind the likelihood approach is to identify all parameters, except of one remaining at a fixed value and save the corresponding objective value. In order to explore the parameter space within certain 

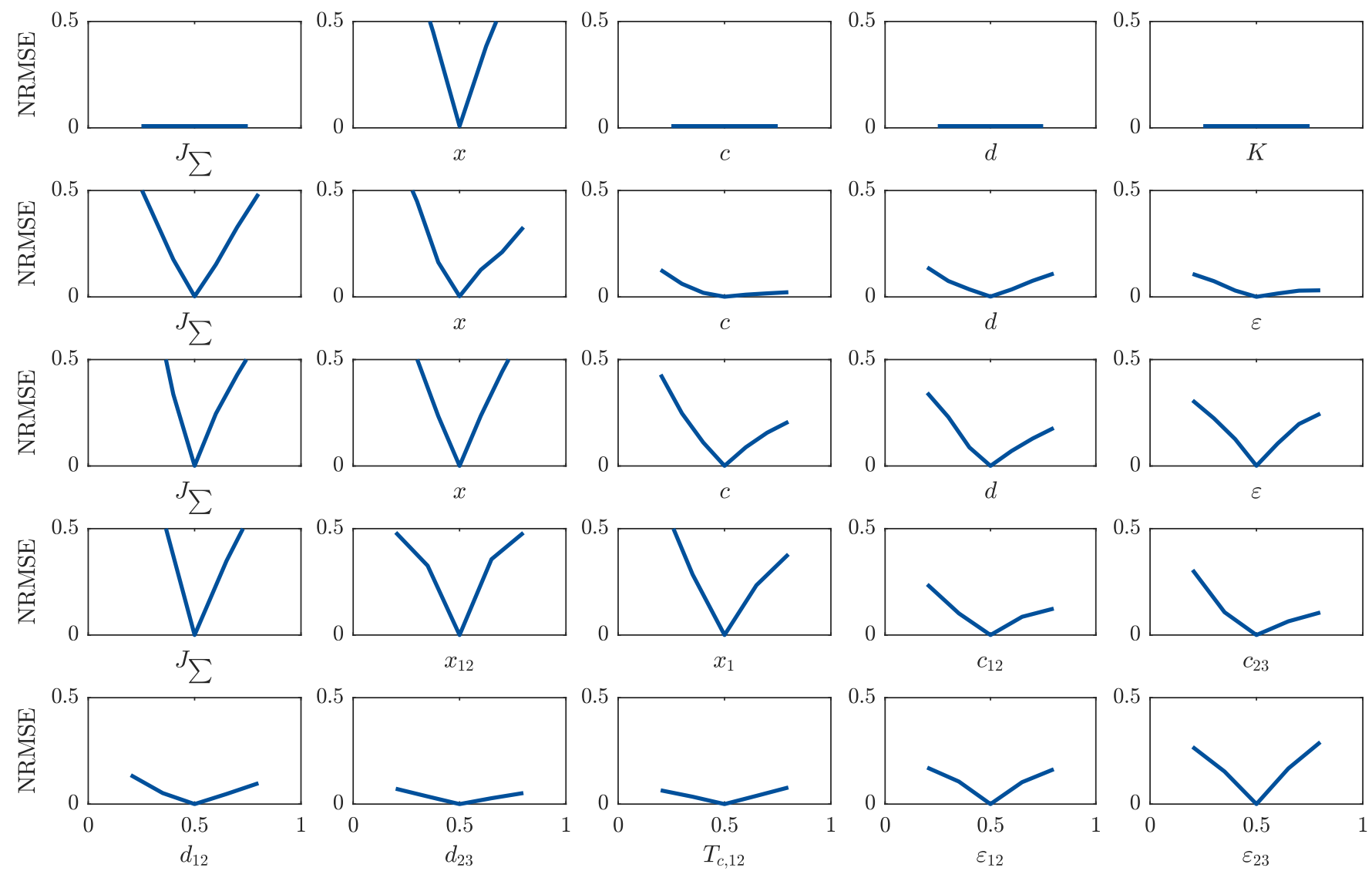

$c_{23}$

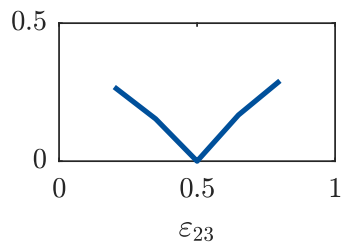

Fig. 5: Likelihood profiles according to the identification setups 1-4 beginning with top panel. Setup 4 consists of the fourth and fifth row. Each profile has been evaluated with seven equidistant points. The true values are always at 0.5.

boundaries, this is repeated for a series of values. Evaluating the objective function a profile of each parameter $\theta_{i}$ may reveal two extremes. If the profile has a unique minimum, the parameter is structural identifiable, as the estimated parameters $\hat{\boldsymbol{\theta}}$ converge to the real parameters $\boldsymbol{\theta}_{0}$. A perfectly flat profile is given in case of ambiguous parameters, so that the corresponding objective function is minimal for any fixed parameter values, as long as the other parameters do not reach their limits. Practically non-identifiable parameters have a unique minimum, but they do not cross a threshold for a desired confidence level of a $\chi^{2}$-distribution. However in this paper only structural identifiability is investigated. In order to neglect the influence of the quality of the measurement data, a simulation model with arbitrary parameter set is used to obtain reference data. Each parameter is assigned to limits, that are $\pm 100 \%$ of the true value. Thus, they can be zero. Since the parameters have different dimensions, they are normalized to a range of $[0,1]$. In order to calculate the likelihood profiles, seven equidistant points are determined. Each identification starts with randomized parameter set. Four different identification setups are analyzed:

1) Two mass system without backlash, using one testsignal, $\boldsymbol{\theta}=\left\{J_{\sum}, x, c, d, K\right\}$

2) Two mass system considering backlash, using one testsignal, $\boldsymbol{\theta}=\left\{J_{\sum}, x, c, d, \varepsilon\right\}$
3) Two mass system considering backlash, using two testsignals, $\boldsymbol{\theta}=\left\{J_{\sum}, x, c, d, \varepsilon\right\}$

4) Three mass system (see Fig. 2), using two test-signals, $\boldsymbol{\theta}=\left\{J_{\sum}, x_{1}, x_{12}, c_{12}, c_{23}, d_{12}, d_{23}, T_{c, 12}, \varepsilon_{12}, \varepsilon_{23}\right\}$, with $x_{1}=\frac{J_{1}}{J_{1}+J_{2}}, x_{12}=\frac{J_{1}+J_{2}}{J_{1}+J_{2}+J_{3}}$.

Regarding setup 1 in Fig. 5 the approach confirms the results from section IV. The profiles of the parameters $\boldsymbol{\theta}=\left\{J_{\sum}, c, d, K\right\}$ show a flat shape. Parameter $x$, on the other hand, has a clear minimum. In identification setup 2 the parameters $\boldsymbol{\theta}=\{c, \varepsilon\}$ are of particular interest. Their profiles indicate a minimum, however, the shape is rather flat, especially in increasing direction. The reason for this asymmetry is that for larger values of backlash a corresponding $c$ can be found, that produces a very similar frequency response as the correct parameter set. Small values of $\varepsilon$ show a linear model behavior that cannot mimic the nonlinear measurement properly, which is revealed by the decreasing side of the profiles. Repeating this setup with two different measurements according to setup 3, parameters $c$ and $\varepsilon$ depict a significantly steeper profile on both sides. Regarding setup 4 parameters $c_{12}, c_{23}, \varepsilon_{12}$ and $\varepsilon_{23}$ are well distinguishable. The damping parameters have a lower gradient, but still show a unique minimum. A discussion about transferability of these results on real measurement data is given in the next section. 


\section{EXPERIMENTAL RESULTS}

In this section the results for the drivetrain parameters according to eq. (6) are presented. The engine dynamics is assumed to be independent from excitation frequency and simplified to $K$ as described in section IV. It forms the excitation signal and hence affects the frequency response. But in reference to the results of section IV, $K$ must not be estimated along with the drivetrain parameters. Hence it is obtained separately using the mean of the values of $\left|G_{\mathrm{ENG}}\right|$ (see eq. (15)) and is expressed as follows

$$
K=\frac{1}{N} \sum_{i=1}^{N}\left|G_{\mathrm{ENG}}\left(\omega_{i}, \boldsymbol{\theta}\right)\right| .
$$

The corresponding results of the frequency response are illustrated in Fig. 6. The test-signals with $A_{\text {test }}=5 \mathrm{Nm}$ and $A_{\text {test }}=10 \mathrm{Nm}$ have been selected for identification (see upper panel) and the other for validation. An overall accurate fit between simulated and real measurement data can be determined. However the graph referred to $A_{\text {test }}=$ $2 \mathrm{Nm}$ indicates most deviations and some irregular shape at $2 \mathrm{~Hz}-5 \mathrm{~Hz}$. This is due to the very low excitation resulting in a low signal-to-noise ratio. Hence any disturbances effect the measurements more significant. Furthermore it has to be noticed, that for real measurements such case of a perfect fit is not achievable for example due to measurement noise, disturbance and model reduction. Hence the cost function is greater than zero as in the presented case. Under this condition the profiles given in Fig. 5 are rounded or even flat at certain error level. So an assignment is only possible within a value range of the flat region.

\section{CONCLUSIONS}

In this paper a frequency based identification approach has been presented in order to obtain physical parameters of the given nonlinear drivetrain model using standard sensors. Within an identifiability analysis of the model it could be

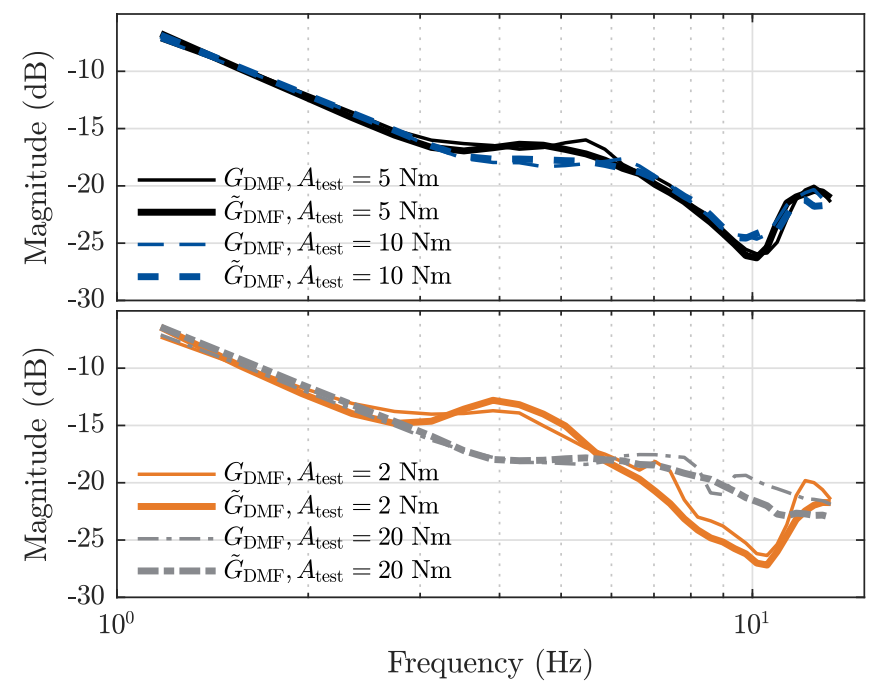

Fig. 6: Measured and identified frequency responses. The thin lines represent real measure data of the vehicle. verified, that the estimation provides unique parameters, considering certain conditions. First is, that the engine dynamics, expressed by a constant gain parameter $K$, cannot be estimated along with the drivetrain parameters. In case of backlash, the corresponding width $\varepsilon$ correlates with the stiffness coefficient $c$, which may lead to ambiguous results according to the measurement quality and model accuracy. As presented a significant improvement is achieved using at least two measurements with different excitation amplitudes. Finally the method has been applied to real measurement data, which show a sufficient agreement with the presented model.

\section{REFERENCES}

[1] M. Grotjahn, L. Quernheim, and S. Zemke, "Modelling and identification of car driveline dynamics for anti-jerk controller design," in 2006 IEEE International Conference on Mechatronics, July 2006, pp. 131-136.

[2] R. Isermann, "Model-based fault-detection and diagnosis status and applications," Annual Reviews in Control, vol. 29, no. 1, pp. $71-85$, 2005.

[3] J. Yang, J. Na, Y. Guo, and X. Wu, "Adaptive estimation of road gradient and vehicle parameters for vehicular systems," IET Control Theory Applications, vol. 9, no. 6, pp. 935-943, 2015.

[4] M. Wielitzka, M. Dagen, and T. Ortmaier, "State and maximum friction coefficient estimation in vehicle dynamics using ukf," in 2017 American Control Conference (ACC), May 2017, pp. 4322-4327.

[5] U. Schaper, O. Sawodny, T. Mahl, and U. Blessing, "Modeling and torque estimation of an automotive dual mass flywheel," in 2009 American Control Conference, June 2009, pp. 1207-1212.

[6] A. Walter, Regelung und Diagnose von Fahrzeug-Antriebssträngen mit Zweimassenschwungrad. Shaker, 2008.

[7] S. Villwock and M. Pacas, "Time-Domain Identification Method for Detecting Mechanical Backlash in Electrical Drives," IEEE Transactions on Industrial Electronics, vol. 56, no. 2, pp. 568-573, 2009.

[8] M. Mola, A. Khayatian, and M. Dehghani, "Identification and adaptive position control of two mass systems with unknown backlash," in 2013 21 st Iranian Conference on Electrical Engineering (ICEE), May 2013, pp. $1-6$.

[9] F. Schütte, Automatisierte Reglerinbetriebnahme für elektrische Antriebe mit schwingungsfäiger Mechanik. Shaker, 2002.

[10] S. Villwock, Identifikationsmethoden für die automatisierte Inbetriebnahme und Zustandsüberwachung elektrischer Antriebe, 2007.

[11] M. Birk, P. Skala, J. Palmer, W. Gerwing, R. Fehrmann, and M. Kessler, "Method for ascertaining individual-cylinder rotation parameters of a shaft of an internal combustion engine," patent US 20090183 559A1, 2009.

[12] M. Mitschke and H. Wallentowitz, Dynamik der Kraftfahrzeuge. Wiesbaden: Springer Fachmedien Wiesbaden, 2014.

[13] L. Guzzella and C. Onder, Introduction to Modeling and Control of Internal Combustion Engine Systems. Springer, 012010.

[14] M. Y. Wang, W. Zhao, and R. Manoj, "Numerical modelling and analysis of automotive transmission rattle," Modal Analysis, vol. 8, no. 7, pp. 921-943, 2002.

[15] M. Nordin, J. Galic', and P.-O. Gutman, "New models for backlash and gear play," International Journal of Adaptive Control and Signal Processing, vol. 11, no. 1, pp. 49-63, 1998.

[16] M. Werner, Signale und Systeme: Lehr- und Arbeitsbuch mit MATLABÜbungen und Lösungen, 3rd ed., ser. Studium. Wiesbaden: Vieweg + Teubner, 2008.

[17] O. Beucher, Signale und Systeme: Theorie, Simulation, Anwendung. Berlin, Heidelberg: Springer Berlin Heidelberg, 2015.

[18] R. Isermann and M. Münchhof, Identification of Dynamic Systems. Springer Berlin Heidelberg, 2011.

[19] E. Walter, Identifiability of Parametric Models. Pergamon, 1987.

[20] A. Raue, C. Kreutz, T. Maiwald, J. Bachmann, M. Schilling, U. Klingmller, and J. Timmer, "Structural and practical identifiability analysis of partially observed dynamical models by exploiting the profile likelihood," Bioinformatics, vol. 25, no. 15, pp. 1923-1929, 2009.

[21] D. Schröder, Intelligente Verfahren. Springer Berlin Heidelberg, 2010 